\title{
Diagnostic strategies in hemoglobinopathy testing, the role of a reference laboratory in the USA
}

\author{
Jennifer L. Oliveira \\ Consultant, Hematopathology, Co-Director, Metabolic Hematology Laboratory, Department of \\ Laboratory Medicine and Pathology, Mayo Clinic, Rochester, MN, USA
}

\begin{abstract}
Although commonly assessed in the context of microcytosis or sickling syndrome screening, hemoglobin mutations may not be as readily considered as a cause of other symptoms. These include macrocytosis with or without anemia, chronic or episodic hemolysis, neonatal anemia, erythrocytosis, cyanosis/hypoxia and methemoglobinemia/sulfhemoglobinemia. Hemoglobin disorders commonly interfere with the reliability of $\mathrm{Hb} \mathrm{A} 1 \mathrm{c}$ measurement. Because the clinical presentation can be varied and the differential diagnosis broad, a systematic evaluation guided by signs and symptoms can be effective. A tertiary care reference laboratory is particularly challenged by the absence of pertinent clinical history and relevant laboratory findings, and appropriate use of resources in a data vacuum can be problematic. To address these issues, our laboratory has constructed testing panels with a tiered strategy utilizing screening assays that detect the most common causes and reflexing additional assays that assess less common etiologies. See Figure 1. Our testing algorithm panels include a rapid hemoglobin fraction monitoring test, a generic diagnostic hemoglobin electrophoresis profile, and more specific diagnostic evaluations for microcytic anemia, hereditary hemolytic anemia, methemoglobinemia and sufhemoglobinemia and erythrocytosis. Use of these testing strategies has facilitated the identification of rare and complex hemoglobin disorders from a wide variety of ethnic groups, including over 500 distinct named alpha, beta and gamma variants (of which 60+ were novel variants at the time of first detection), 99 beta thalassemia mutations and greater than 20 large deletional beta globin cluster deletion subtypes.
\end{abstract}

\section{Introduction}

Hemoglobinopathy evaluation is undertaken in a wide range of clinical situations which include newborn, prenatal or preconception screening, diagnostic testing to explain specific clinical signs

\author{
Correspondence: Jennifer L. Oliveira, Mayo Clinic, Department of \\ Laboratory Medicine and Pathology, 200 First ST SW, Rochester, MN \\ USA 55902. \\ Tel. (507) 284-1143; Fax (507) 284-5115. \\ E-mail: oliveira.jennifer@mayo.edu \\ Conflict of interest: The author has nothing to disclose \\ This work is licensed under a Creative Commons Attribution 4.0 \\ License (by-nc 4.0). \\ CC Copyright J.L. Oliveira, 2018 \\ Licensee PAGEPress, Italy \\ Thalassemia Reports 2018; 8:7476 \\ doi:10.4081/thal.2018.7476
}

and symptoms, and monitoring of hemoglobin $(\mathrm{Hb})$ fractions after therapy. This varied range of test indications complicates a uniform streamlined approach to hemoglobin testing. Cost effective resource utilization requires knowledgeable application of the varied methods available and their complementary strengths and limitations to select tests best suited to answer the clinical need. In the tertiary reference laboratory setting, understanding the goal of the ordering provider and therefore the optimal extent of testing is challenging. Accurately relating the particular clinical question helps laboratorians process samples appropriately but this simple and basic information is too often not adequately communicated in real testing situations. Because the indications for hemoglobin testing are so wide-ranging, our laboratory approaches hemoglobinopathy testing in a tiered approach. See Figure 2.

\section{Methods and Discussion}

The least complex clinical situation encountered in hemoglobin testing is the monitoring of a previously confirmed hemoglobin disorder. Monitoring is frequently used in sickle cell disorder patients with recent transfusion or hydroxyurea therapy to quantify $\mathrm{Hb} \mathrm{A}, \mathrm{Hb}$ $\mathrm{F}$ and the abnormal variant(s). This can be rapidly accomplished using a single detection method that reliably quantitates the variant percentage. We use high performance liquid chromatography (HPLC) or capillary electrophoresis (CE) for this purpose.

The next level of complexity is a diagnostic generic hemoglobin evaluation. This is a test that carries the most ambiguity regarding the level of testing required to answer a clinical question. It is designed as a diagnostic test which detects all of the most common $\mathrm{Hb}$ variants, quantitates $\mathrm{Hb} \mathrm{A}_{2}$ for the diagnosis of beta thalassemia minor and guides further testing for uncommon hemoglobin disorders. This heavily relies upon adequate communication from referring providers for proper interpretation and utilization management. Communication can be a written information sheet or electronic comment field to relay complete blood count (CBC) values, test indication/clinical suspicion, clinical/family history, ethnicity, and data regarding recent therapeutic confounders such as transfusion or hydroxyurea administration. Our laboratory utilizes HPLC [1] and CE [2] simultaneously as screening methods. If normal tracings are obtained and no further indication is known to be present, the result is released as negative with a caution statement that addresses the types of conditions that further evaluation could be considered. If indicated, additional tests are performed according to the possible identification of the disorder suspected. These can include sickle solubility, isoelectric focusing, hemoglobin stability (heat and isopropanol), flow cytometry for $\mathrm{Hb} \mathrm{F}$ red cell distribution, mass spectrometry (MS) and molecular studies to include DNA sequencing of the alpha, beta and gamma globin genes and MLPA analysis of alpha and beta globin cluster loci. Because many methods are available, judicious use of the testing options requires clinical information and knowledge of the strengths and limitations of the methods available for diagnosis. 
To aid in the diagnosis of very complex hemoglobin disorders, a more comprehensive testing profile is offered in which HPLC and $\mathrm{CE}$ results are correlated with ferritin levels, possible deletional alpha thalassemia testing, and red blood cell (RBC) indices. This allows for incorporating iron deficiency and alpha thalassemia status and assumes an indication for more extensive investigations to explain the phenotype. Multifactorial causes are addressed, such as beta thalassemia trait that shows a more severe phenotype than expected, or complex Hb E disorders. This strategy has identified many electrophoretically silent clinically significant variants $(\mathrm{Hb}$ Adana, $\mathrm{Hb}$ Bronovo, $\mathrm{Hb}$ Taybe) that may be missed by our routine hemoglobin electrophoresis test offering.

Some indications for hemoglobin testing encompass a broader scope of RBC disorders. To address these needs we use panels focused on particular signs and symptoms, in particular, hemolysis, methemoglobinemia, or erythrocytosis. The panel that assays for hereditary causes of hemolysis includes hemoglobin (with frontline stability study testing), RBC enzyme and membrane disorder testing. It incorporates RBC enzyme activities and osmotic fragility/EMA binding assays for membrane disorders correlated with a review of the peripheral blood smear. The addition of the stability study screen identifies many electrophoretically silent unstable $\mathrm{Hb}$ variants. Another panel that focuses on methemoglobinemia and sulfhemoglobinemia performs HPLC and CE, methemoglobin and sulfhemoglobin levels and methemoglobin reductase (METR) [cytochrome b5 reductase 3] activity levels [3]. This provides for the identification of M-hemoglobin variants which were historically challenging to identify using previous methods but have characteristic patterns using the HPLC method. Elevated methemoglobin or sulfhemoglobin values prompt further investigation for a variant that can be subtle or silent by electrophoresis and HPLC, such as Hb Volga. In addition, this profile also assesses for METR deficiency-associated congenital methemoglobinemia.

Our laboratory has found great utility in a panel for the evaluation of hereditary erythrocytosis which includes frontline oxygen dissociation curve (p50) and MS testing [4]. Serum erythropoietin and p50 results efficiently guide reflex testing from the 19 different assays available for erythrocytosis. In our experience, hereditary erythrocytosis is commonly caused by high oxygen affinity hemoglobin variants (HOA) which are associated with a decreased p50 value. Many HOAs have neutral charge substitutions, do not separate from $\mathrm{Hb}$ A using multiple protein screening methods and can result in false negative cases. Simultaneously performing MS, HPLC and CE methods allows for the detection of all of the 79 distinct alpha and beta globin chain HOA variants we have seen in our laboratory (manuscript submitted for publication). In addition, the MS method enables an estimate of variant percentages in many cases that do not separate from $\mathrm{Hb}$ A by other methods. When a HOA is excluded, this panel tests for erythropoietin receptor (EPOR), 2,3-bisphosphogycerate mutase (BPGM) and oxygen sensing pathway mutations (HIF2A, PHD2 and VHL) as possible etiologies for the elevated hematocrit in an algorithmic fashion.

\section{Conclusions}

In summary, indications for the evaluation of hemoglobin disorders vary from the simple to the very complex. Laboratories at tertiary reference centers harbor extensive experience but suffer from a dearth of preanalytical information critical in the accurate interpretation and efficient triaging of appropriate testing for these disorders. Creating specific testing panels based on signs and symptoms allows assignment of implied indications for testing and utilize appropriate algorithms. These testing strategies allow effi- cient diagnoses of rare clinically significant hemoglobin disorders and decrease widespread indiscriminate testing that wastes resources. While continued suboptimal communication between clinical caregivers and laboratorians still result in missed opportu-

\begin{tabular}{|c|c|c|}
\hline & $\begin{array}{l}\text { - Sickling syndromes } \\
\text { - Pain crises, infection risk, etc. } \\
\text { - Microcytosis } \\
\text { - Unnecessary/harmful Fe Rx, eve }\end{array}$ & $\begin{array}{l}\text { Panel focused on } \\
\text { Thalassemia and } \\
\text { Hemoglobinopathy }\end{array}$ \\
\hline & $\begin{array}{l}\text { - Hemolysis/Macrocytosis } \\
\text { - Anemia of variable severity } \\
\text { - Hyperbilirubinemia } \\
\text { - Gallstones/cholecystitis } \\
\text { - Splenomegaly } \\
\text { - Aplastic crises } \\
\text { - Hydrops fetalis } \\
\text { - Erythrocytosis Panel focus }\end{array}$ & $\begin{array}{l}\text { Panel focused on } \\
\text { Hemolytic Anemia }\end{array}$ \\
\hline & $\begin{array}{l}\text { - Cyanosis/Hypoxia, benign } \\
\text { - Unnecessary evals/Rx } \\
\text { - Met/sulfhemoglobinemia }\end{array}$ & $\begin{array}{l}\text { Panel focused on } \\
\text { Methemoglobinemia } \\
\text { Sulfhemoglobinemia }\end{array}$ \\
\hline
\end{tabular}

Figure 1. Hemoglobin disorders should be considered in settings other than sickling syndromes or microcytic anemia. Because hemoglobin testing includes a wide range of clinical indications ranging from monitoring of known variants, to low suspicion prenatal screening to high suspicion complex disorders, adequate communication of preanalytical data is crucial for proper and efficient testing of specimens. Creating specific assay panels based on signs and symptoms allows appropriate test utilization guided by useful algorithms.

\section{$\mathrm{Hb}$ Testing Strategy}

$\begin{aligned} \begin{array}{r}\text { Protein analysis } \\ \text { First line Screening } \\ \text { HPLC }\end{array} & \begin{array}{l}\text { Molecular Analysis } \\ \text { Second or Third line testing } \\ \text { Alpha globin cluster locus del/dup }\end{array} \\ \begin{array}{r}\text { Capillary electrophoresis } \\ \text { Beta globin cluster locus del/dup } \\ \text { Second line reflex testing } \\ \text { Isoelectric focusing }\end{array} & \text { HBB gene sequencing } \\ \text { Mass spectrometry, intact } & \\ \text { Sickle solubility } & \\ \text { F distribution flow cytometry } & \\ \text { Heat stability } & \\ \text { Isopropanol stability } & \end{aligned}$

Figure 2. Hemoglobin testing strategy in a high throughput testing laboratory in the United States of America. To increase sensitivity, two screening methods are used for all diagnostic assays. Dependent upon the choice of testing ordered, additional methods may be used a frontline screening methods, such as stability studies for hemolysis, $\mathrm{p} 50$ assay for erythrocytosis and methemoglobin and sulfhemoglobin levels for cyanosis. An assay for monitoring previously confirmed hemoglobin disorders may use a single test that reliably separates and quantitates common hemoglobin fractions. 
nities to identify all clinically significant variants, the use of these testing strategies in our laboratory has facilitated the identification of rare and complex hemoglobin disorders from a wide variety of ethnic groups, including over 500 distinct named alpha, beta and gamma variants (of which 60+ were novel variants at the time of first detection), 99 beta thalassemia mutations and greater than 20 large deletional beta globin cluster deletion subtypes [4-10].

\section{References}

1. Szuberski J, Oliveira JL, Hoyer JD. A comprehensive analysis of hemoglobin variants by high-performance liquid chromatography (HPLC). Int J Lab Hematol 2012 Dec; 34(6): 594-604.

2. Riou J, Szuberski J, Godart C, Wajcman H, Oliveira JL, Hoyer JD, Bardakdjian-Michau J. Precision of CAPILLARYS 2 for the Detection of Hemoglobin Variants Based on Their Migration Positions. Am J Clin Pathol. 2018 Jan 29;149 (2):172-180. PMID: 29365076.

3. Oliveira JL, Rangan A, Coon L, Hein MS, Savedra ME, Swanson KC, Szuberski J, Nguyen PL, Go RS, Hoyer JD. MHemoglobin variants with associated Methemoglobin and Sulfhemoglobin levels and Methemoglobin Reductase Activity. ISLH, Honolulu, HI, USA, May 2017.

4. Oliveira JL, Frederick LA, Coon L, Hein MS, Grebe SK, Patnaik MM, Pardanani A, Tefferi A, Viswanatha DS, Hoyer
JD. Spectrum of Mutations Associated with Hereditary Erythrocytosis. Blood 2015 126:2140.

5. Fairbanks, VF, et al., Familial erythrocytosis due to electrophoretically undetectable hemoglobin with impaired oxygen dissociation (hemoglobin Malmo, alpha 2 beta $297 \mathrm{gln}$ ). Mayo Clin Proc, 1971. 46(11): p. 721-7.

6. Hoyer JD, Wendt PC, Hogan WJ, Oliveira JL. Hb Nebraska [ $\beta 86$ (F2)Ala $\rightarrow$ Ile (HBB:c.259G $>$ A;260C $>$ T) ]: A Unique High Oxygen Affinity Hemoglobin Variant with a Double Nucleotide Substitution within the Same Codon. Hemoglobin. 2011;35(1):22-7.

7. Hoyer, JD, et al., $\mathrm{Hb}$ Tak confirmed by DNA analysis: not expressed as thalassemia in a $\mathrm{Hb} \mathrm{Tak} / \mathrm{Hb} \mathrm{E}$ compound heterozygote. Hemoglobin, 1998. 22(1): p. 45-52.

8. Inoue S, Oliveira JL, Hoyer JD, Sharman M. Symptomatic Erythrocytosis Associated with a Compound Heterozygosity for $\mathrm{Hb}$ Lepore-Boston-Washington (887- $\beta 116)$ and Hb Johnstown [ $\beta 109(G 11)$ Val $\rightarrow$ Leu, GTG>TTG]. Hemoglobin. 2012 May 7.

9. Oliveira, JL, et al., Hb Cambridge-MA [beta144(HC1)beta146(HC3)Lys-Tyr-His-->0 (HBB c.433 A>T)]: a new high oxygen affinity variant. Hemoglobin, 2010. 34(6): p. 565-71.

10. Hein, MS, Oliveira, JL, Swanson, KC, Lundquist, PA, Yungerberg, JA, Coon, LM, Dawson, BD, Go, RS, Jevremovic, D, and Hoyer, JD (2015). Large Deletions Involving the Beta Globin Gene Complex: GenotypePhenotype Correlation of 119 Cases. Blood, 126(23), 3374. 